\title{
INTERAÇAO PRECOCE MAE-BEBÊ E A CONCEPÇÃO DO DESENVOLVIMENTO INFANTIL INICIAL*
}

\author{
EARLY MOTHER-CHILD INTERACTION AND THE \\ CONCEPTION OF CHILD DEVELOPMENT
}

\author{
Maria Lucia Seidl de Moura ${ }^{1}$ \\ Adriana F. P. Ribas ${ }^{2}$
}

SEIDL de MOURA, M.L.; RIBAS, A.F.P. A Interação Precoce Mae-Bebê e a Concepção do Desenvolvimento Infantil Inicial. Rev. Bras. Cresc. Desenv. Hum., São Paulo, 8(1/2), 1998.

Resumo: Este trabalho integra uma linha de investigações que tem tentado articular a perspectiva sócio-cultural com outras vertentes de investigação: a do desenvolvimento infantil inicial e a das interações adulto-bebe Foram identificados elementos que constituem os contextos de interação mãe-bebê (e. g. cenários , atividades , episódios de interação e tentativa de interação e representações) e investigadas relações entre as concepções de mães acerca das competências dos bebes e a natureza de suas atividades e interações com eles. Q uir^Lze díades mãe-bebe recém-nascido foram filmadas durante 20 minutos em suas residências. Foram utilizados uma metodologia de observação e um questionário (QCBR) desenvolvidos pelo próprio grupo de pesquisa. Foi identificada correlação significativa entre o índice de atividade da mãe e o escore total no QCBR. Não foi identificada correlação entre o escore total no QCBR e a variável interação. Tais resultados coníribuem para uma melhor compreensão do desenvolvimento cognitivo como processo inseparável do contexto social.

Palavras-chave: interação mãe-bebê; desenvolvimento infantil inicial; concepção do desenvolvimento.

\section{INTRODUÇÃO}

Este trabalho dá continuidade a investigações sobre o papel da interação social no desenvolvimento cognitivo (RIBAS, 1993; RIBAS, 1996; RIBAS \& SEIDL DE MOURA, 1995; SEIDL DE MOURA, 1995), buscando uma melhor compreensão do desenvolvimento cognitivo como um processo que se dá no contexto social.
Em nossa espécie, o recém-nascido é completamente dependente de adultos para sua sobrevivência. Esses adultos, por sua vez, como membros da cultura, recebem o recém-nascido com expectativas, concepções e práticas que vão colocar limites, organizar e/ou facilitar o desenvolvimento deste novo membro do grupo social (COLE, 1996). Este autor, discutindo como se dão as transformações nas interações de mentes do

\footnotetext{
* Trabalho realizado a partir dos dados parciais da pesquisa coordenada pela primeira autora. Participaram da pesquisa: Rodolfo de C. Ribas Junior, professor do instituto de Psicologia da UFRJ; Gabriela G. Albennaz; Guilhennc de Carvalho; Karla da C. Seabra; Luciana F. Pessôa; Soraya C. M Oliveira; Susana E. Nogueira; e Thais Monteiro Koloff, alunos de graduação em Psicologia e bolsistas de iniciação científica UERJ, FAPERJ e CNPq. Dados parciais desta pesquisa foram apresentados no XXV1 Congresso Interamericano de Psicologia, São Paulo, 1997 e no II Congresso Brasileiro de Psicologia do Desenvolvhnento, Gramado, 1998.

1 Coordenadora do Curso de Mestrado em Psicologia da Universidade do Estado do Rio de J aneiro. Endereço para conrespondência: Rua Fritz Feigl, 465, Eldorado, Rio de Janeiro, Brasil . E-mail: mlseid1@ ax.apc.org. Universidade Federal do Rio de Janeiro, Pós-Graduação em Psicologia Social. Rua São Francisco Xavier, 524. 10ªndar Bloco F / Sala 10.019 Maracanã, Rio de Janeiro, RJ. CEP 20559-9000.

2. Psicóloga, Mestre em Psicologia. Bolsista de Apoio Técnico FAPERJ. E-mail: aribas@ax.apc.org.
} 
nascimento à velhice, aponta como a contribuição dos pais na interação é mediada pela cultura: "Seus processos interpretativos desempenham um papel controlador na interação e se se pode dizer que um encontro de mentes ocorreu ou não. Para os pais, um bebê não é um objeto 'natural', destituído de sentido. Eles interpretam as propriedades do bebê em termos de categorias culturais existentes" (p. 67).

BRUNER (1996) observa que a partir dos estudos de privação em animais de laboratório, se passou a estudar de forma sistemática e controlada o desenvolvimento de bebês humanos em termos de sua percepção, atenção, memória, imitação e ação (KALINS \& BRUNER, 1973; PAPOUSEK, 1979). Esta literatura tem crescido desde a década de sessenta e um corpo significativo de evidências tem apontado para as capacidades dos recém-nascidos de perceber o mundo, de imitação de comunicação e de realizar ações reguladas por informação perceptual (BRUNER, 1983; TREVARTHEN \& HUBLEY, 1978; BERTENTHAI, 1996). Entre outros aspectos, tem sido verificado que a percepção de objetos e seu reconhecimento começa no nascimento (BERTENHAI, op. cit.). Este autor, em revisão recente, conclui que: "Recém-nascidos são dotados com muito mais do que reflexos ou padrões fixos de ação. Suas ações são dirigidas a metas e coordenadas especialmente, embora lhes falte consistência e sejam frequentemente confundidas por outros fatores, tais como os de postura. Além disso, os recém-nascidos mostram memória de reconhecimento desde o nascimento e em torno de três meses os bebês raciocinam sobre eventos que estão parcialmente ocultos” (p. 452-453).

Como afirma Bruner, "Os bebês, afinal, se mostraram muito mais espertos, mais proativos do que reativos, mais atentos ao mundo social imediato ao seu redor do que se suspeitava anteriormente. Eles realmente não habitavam um mundo de barulhenta e ‘nebulosa confusão’: eles pareciam em busca de estabilidade preditiva desde o início” (BRUNER, 1996, p. 72).

Neste aparato básico se distingue um conjunto de características que parecem especialmente preparar o bebê para a interação com os outros, como sua capacidade de distinguir configurações de faces humanas, sua preferência por esses tipo de configuração e por sons de vozes humanas, sua capacidade de reconhecer a voz materna e o cheiro de seu leite, entre outros aspectos (KLAUS \& KLAUS, 1989).
Paralelamente aos estudos que produziram estas evidências, investigações sobre trocas entre bebês e adultos têm identificado a presença de interações precoces e estudado suas características, sobretudo em díades mãe-bebê. Os resultados desses estudos têm contribuído para o abandono de modelos unidirecionais, que se centram nas influências que os adultos exercem sobre as crianças, e para a adoção de modelos bidirecionais, caracterizados pela ênfase na reciprocidade e na adaptação mútua entre os parceiros. Sob esta última perspectiva, tem sido identificada a atribuição, por parte de mães, de significado e intenção aos comportamentos do bebê e o estabelecimento da atenção conjunta desde muito cedo (SCAIFE \& BRUNER, 1975; BORNSTEIN \& TAMIS-LEMONDA, 1990). Em síntese, como diz BRUNER (1996), “o que esses estudos revelaram foi a importância da interação humana de mão-dupla” (p.72). Ou seja, não mais se pensa em um bebê que apenas é estimulado pelas ações dos adultos, mas em trocas de parceiros de capacidades distintas, mas igualmente ativos.

No grupo de pesquisa do qual fazem parte as autoras deste trabalho tem-se procurado articular a perspectiva teórica sócio-interacionista ou sócio-cultural (WERTSCH, DEL RIO \& ALVAREZ, 1995), centrada no estudo do desenvolvimento cognitivo como um processo social, a essas duas vertentes de investigação, a do desenvolvimento infantil inicial (infant development) e a das interações iniciais mãe-bebê, que convergem e se integram, embora não totalmente. Neste sentido, esforços têm sido despendidos com o objetivo de aprimorar a noção de zona de construção (NEWMAN, GRIFFIN \& COLE, 1989) e de caracterizar interações mãe-bebê como zonas de construção.

Considera-se ainda, tal como COLE (1996), que a abordagem sócio-interacionista ou sócio-cultural é compatível com a perspectiva de BALTES e STAUDINGER (1996) sobre a interação no curso do desenvolvimento. Para estes, a expressão, mentes interativas é uma metáfora interessante para discutir o papel da interação social na evolução e ontogênese da mente, e visa comunicar que "a interação entre mentes é fundamental para a compreensão psicológica da cognição, tanto em Psicologia Cognitiva, como do Desenvolvimento” (p. 7). Sua caracterização desta metáfora inclui neste território conceitual transações externas e internas, com representações mentais de outras pessoas.

3 Nessa e nas citações seguintes a tradução é das autoras. 
Por fim, tem-se tentando utilizar os conceitos de formatos (BRUNER, 1983), scripts e contextos (COLE, 1996) na caracterização das interações precoces mãe-bebê. Formatos são entendidos como scripts básicos e inatos como a capacidade dos parceiros regularem a atenção conjuntamente e o processo de afiliaçao. Scripts são conjuntos de atividades que tendem a ser repetidas, em contextos específicos de ação e que são criados culturalmente. O entendimento é de que em cada contexto de inter-ação, os parceiros mobilizam scripts básicos, como a regulação conjunta da atenção e o processo de afiliação e também scripts construídos culturalmente.

O esforço de integração teórica e de evidências empíricas que tem sido realizado nas investigações feitas inclui, além das vertentes já citadas, o estudo de concepções de adultos sobre desenvolvimento infantil. Levando-se em conta que a mãe, ao interagir com o bebé, se utiliza de um inventário próprio de representações e expectativas acerca do mundo, que incluem representações e expectativas sobre os próprios bebés, impõe-se também examinar o papel destas concepções no estabelecimento de interações mãesbebês e no próprio desenvolvimento.

De fato, a investigação das concepções de pais e outros adultos acerca do processo de desenvolvimento humano caracteriza hoje uma importante área de pesquisa em Psicologia do Desenvolvimento. A natureza de tais concepções, sua influência sobre o comportamento dos adultos e suas possíveis relações com o desenvolvimento infantil têm sido objeto de investigação, como se pode observar nas revisões apresentadas por GOODNOW (1988) e MILLER (1988), sendo também objeto de estudo desta pesquisa.

Em uma fase inicial das investigações, foi desenvolvido um instrumento (RIBAS \& SEIDL DE MOURA, 1995) para a avaliação da concepção de adultos acerca das competências do bebê recém-nascido. Este instrumento (QCBR) foi utilizado na investigação de variáveis associadas à concepção que os adultos têm dos bebês (SEIDL DE MOURA, RIBAS \& RIBAS ${ }^{4}$ ). A análise revelou um efeito significativo das variáveis sexo e escolaridade. As mulheres na amostra estudada apresentaram uma média no QCBR que indica uma concepção do bebê recém-nascido como capaz de discriminações, preferências e dotado de capacidades perceptivas complexas que o tornam sensível às interações. Aparentemente, este tipo de conhecimento parece ser considerado como do domínio das mulheres que, assim, seriam mais sensíveis e atentas às informações acerca do desenvolvimento e das características do bebé recém-nascido.

Finalmente, foi desenvolvida uma metodologia de observação e análise de interação de díades mãe-bebê, que será descrita posteriormente. Esta metodologia foi utilizada em uma pesquisa longitudinal (RIBAS, 1996) na qual foram realizadas quatro observações de uma díade mãebebê ao longo de cinco meses.

Com base nas investigações já conduzidas pelo próprio grupo de pesquisa e dando continuidade aos trabalhos já citados, com o mesmo objetivo geral de investigar a gênese de zonas de construção, e a constituição de mentes interativas, este estudo tem como objetivos específicos: analisar a natureza das interações mãe-bebê no primeiro mês de vida do bebê; analisar diferenças nessas interações relacionadas à concepção das mães sobre o desenvolvimento infantil inicial; organizar um acervo de registros em vidro de díades mãe-bebê.

\section{MÉTODO}

\section{Amostra}

A amostra total desta pesquisa será constituída por vinte díades mãe-bebê. Até o momento, 15 díades foram observadas e seus dados são analisados e discutidos neste trabalho.

Todos os bebês observados nasceram a termo e não necessitaram de cuidados médicos especiais. Sua idade na ocasião da coleta de dados variou entre 22 e 37 dias, sendo a idade média 28 dias. A idade das mães variou entre 19 e 39 anos, sendo a idade média 28 anos. Do total de quinze mães, dez foram observadas com seu primeiro filho, quatro com o segundo filho e uma com o terceiro. Realizavam uma atividade profissional fora do lar $60 \%$ das mães observadas e $40 \%$ trabalhavam exclusivamente em casa. Seu nível de instrução foi predominantemente de segundo e terceiro graus. As díades residiam em bairros de classe média urbana do Estado do Rio de Janeiro.

\section{Instrumento \\ Concepção sobre o desenvolvimento infantil inicial}

Foi aplicado às mães o Questionário sobre a Concepção de Competências do Bebê Recém-

4 SEIDL DE MOURA, M. L.; RIBAS, A. F. P. \& RIBAS, R. C. Brazilian Adults ‘conceptions of Newborn Babis’ competence’. Artigo submetido para publicação. 
nascido ( $Q C B R$, 1F Guttman split-half $=0,86)$, que inclui 35 itens (RIBAS \& SEIDI DE MOURA, 1995).

\section{Metodologia para observação e análise da interação de díades mãe-bebê}

A metodologia de observação e análise de interação de díades mãe-bebê foi desenvolvida pelo grupo de pesquisa (SEIDL DE MOURA \& RIBAS, 1996) a partir de categorias propostas por BORNSTEIN e TAMIS LEMONDA ( 1990), VIBBERT e BORNSTEIN (1989) e ISABELLA, BELSKY e EYE (1989). São incluídas categorias de observação divididas em grupos de observação de interação, observação de atividade (da mãe e do bebê) além de estados do bebê (BRAZELTON CRAMER, 1992).

As categorias de observação de interacão são: domínio social de interação (DS), domínio didático de interação (DD) e tentativa da interação não efetivada (T). As de observação de atividade da mãe são: gostos $(G)$, vocalizações $(V)$, fala (F), atribuição de significado (AS), sorrir (S), toque (T), olhar o bebê (OB), mostrar objeto (MO), cantar (CA) e pegar no colo (PC). As de observação de atividade do bebê são: olhar o ambiente (OA), olhar a mãe (OM), movimentar membros (MM), tocar a mãe (TM), mamar (M), pegar objeto (PO), vocalizar (V), sorrir (S) e fechar os olhos (FO). Juntamente com as diversas categorias foram registrados os contextos de troca da díade.

Foram classificados ainda os estados de vigília do bebê, considerando os 30 intervalos de observação, utilizando-se a classificação proposta inicialmente por WOLFF em 1966 (conforme citado por LEBOVICI, 1987), extremamente similar à classificação proposta por BRAZELTON \& CRAMER (1992). Tal classificação inclui seis estados, que variam do sono profundo (estado 1) ao choro (estado 6 ).

\section{Procedimento}

Os dados foram colhidos durante a interação da mãe com seu bebê, em ambiente natural, sem interferência deliberada do observador. Foi realizado o registro em vídeo das atividades da mãe e do bebê, iniciado após 10 minutos de familiarização com o observador e durante 20 minutos. Além do registro em vídeo, o observador registrou aspectos gerais da situação e acontecimentos relevantes. Após a realização da filmagem, a mãe respondeu ao $Q C B R$.

\section{Levantamento de Dados}

Os dados obtidos com o QCBR foram levantados e transferidos para um banco de da- dos. Dos vinte minutos de vídeo, realizados em cada uma das observações, foram desconsiderados cinco minutos, sendo analisados os 15 minutos restantes, em intervalos iguais de 30 segundos. Os dados foram levantados através do preenchimento da Folha de Registro de Interação e Atividades na qual foram registradas as ocorrências de atividades da mãe e do bebê nos intervalos e registradas sequências de atividade conjunta, consideradas como instâncias de interação. Foram examinados, ainda, os contextos de troca em cada um dos intervalos e transcritas todas as falas e vocalizações da díade durante a observação. Para cada um dos intervalos de observação foi classificado também o estado de vigília do bebê. Para cada bebê foi realizado um levantamento dos diferentes estados encontrados, em termos de percentagem de ocorrência de intervalos, e foi identificado o estado predominante do bebê durante a observação.

\section{RESULTADOS E DISCUSSÃO}

Os dados de cada díade foram analisados em termos da percentagem de ocorrência das atividades da mãe e do bebê e das ocorrências de interação e tentativa de interação em relação ao número total de intervalos de observação. As atividades realizadas pela mãe e pelo bebê nos 30 intervalos foram comparadas e uma análise qualitativa destas atividades foi realizada.

Os episódios de interação identificados foram analisados em termos da sua descrição, contexto, quadro descritivo, duração e sincronia interacional. Uma análise qualitativa das interações levou em consideração aspectos como: natureza das atividades dos parceiros, ocorrência ou não de ajustes, por parte da mãe, no sentido de favorecer interações, cenários mais frequentes de ocorrência de interação, entre outros.

Os dados relativos às percentagens de ocorrência de atividades das mães e bebês e ocorrências de interação são apresentados na Tabela 1.

Como se pode verificar, as atividades predominantes das mães foram: Olhar o bebê $(99,8 \%)$ e tocar o bebê $(85,6 \%)$. O predomínio da atividade de olhar o bebê foi encontrado também por KAYE e FOGEL ( 1980), em uma pesquisa longitudinal na qual os autores relatam que mães de bebês de 6, 13 e 26 semanas despendiam cerca de $100 \%$ do tempo de observação olhando os bebês diretamente. O toque também foi apontado por estes autores como atividade crucial das mães no sentido de atrair a atenção dos bebês. Estes achados sugerem a importância das atividades de olhar e tocar no processo interacional 
Tabela1: Ocorrência de Interação e Atividade

\begin{tabular}{|c|c|c|c|c|c|c|c|c|c|c|c|c|c|c|c|c|c|}
\hline & & & & & & & & & & Díade & & & & & & & \\
\hline & & 01 & 02 & 03 & 04 & 05 & 06 & 07 & 08 & 09 & 10 & 11 & 12 & 13 & 14 & $4 \quad 15$ & Média \\
\hline & DS & 10 & 3 & 3 & 20 & 10 & 13 & 0 & 0 & 7 & 17 & 0 & 10 & 7 & 7 & $7 \quad 17$ & 8,2 \\
\hline I & DD & 0 & 0 & 0 & 0 & 0 & 0 & 0 & 0 & 0 & 0 & 0 & 0 & 7 & 0 & 0 & 0.4 \\
\hline & $\mathrm{T}$ & 7 & 10 & 0 & 0 & 0 & 0 & 0 & 0 & 0 & 0 & 0 & 0 & 23 & 0 & 0 & 2,7 \\
\hline & G & 3 & 0 & 0 & 0 & 0 & 0 & 0 & 0 & 0 & 0 & 0 & 0 & 0 & 0 & 0 & 0,2 \\
\hline & $\mathrm{V}$ & 10 & 50 & 3 & 13 & 0 & 27 & 7 & 0 & 10 & 7 & 37 & 37 & 33 & 0 & $\begin{array}{ll}0 & 13\end{array}$ & 16,4 \\
\hline & $\mathrm{F}$ & 100 & 83 & 27 & 83 & 80 & 20 & 77 & 37 & 73 & 100 & 93 & 57 & 93 & 100 & $\begin{array}{l}0 \quad 57 \\
0\end{array}$ & 72,0 \\
\hline & S & 10 & 0 & 17 & 13 & 7 & 3 & 0 & 0 & 0 & 37 & 13 & 3 & 20 & 40 & $\begin{array}{l}0 \quad 23 \\
\end{array}$ & 12,4 \\
\hline AM & $\mathrm{T}$ & 100 & 90 & 100 & 87 & 47 & 100 & 97 & 90 & 83 & 90 & 100 & 100 & 100 & 97 & 3 & 85,6 \\
\hline & OB & 100 & 100 & 100 & 100 & 100 & 100 & 100 & 100 & 100 & 100 & 100 & 100 & 100 & 97 & 7100 & 99,8 \\
\hline & $\mathrm{MO}$ & 0 & 17 & 0 & 0 & 0 & 0 & 0 & 0 & 0 & 3 & 0 & 0 & 7 & 0 & 0 & 1,8 \\
\hline & $\mathrm{Ca}$ & 0 & 3 & 0 & 3 & 0 & 3 & 0 & 0 & 0 & 3 & 3 & 0 & 0 & 0 & 0 & 1,1 \\
\hline & PC & 7 & 3 & 3 & 3 & 17 & 3 & 3 & 7 & 0 & 13 & 7 & 10 & 20 & 13 & 0 & 7,3 \\
\hline & AS & 33 & 23 & 10 & 13 & 27 & 7 & 20 & 7 & 23 & 47 & 17 & 3 & 30 & 30 & $\begin{array}{ll}0 \quad 37\end{array}$ & 21,8 \\
\hline & $\mathrm{OA}$ & 73 & 90 & 50 & 83 & 53 & 50 & 47 & 70 & 80 & 60 & 20 & 67 & 100 & 93 & $\begin{array}{l}3 \quad 30\end{array}$ & 64,4 \\
\hline & $\mathrm{OM}$ & 43 & 40 & 10 & 50 & 57 & 13 & 47 & 47 & 37 & 57 & 10 & 27 & 10 & 10 & $\begin{array}{ll}0 \quad 27\end{array}$ & 32,2 \\
\hline & $\mathrm{MM}$ & 177 & 90 & 87 & 77 & 93 & 100 & 93 & 77 & 97 & 100 & 93 & 93 & 93 & 93 & $\begin{array}{ll}3 \quad 83\end{array}$ & 89,8 \\
\hline $\mathrm{AB}$ & $\mathrm{TM}$ & 3 & 67 & 7 & 47 & 23 & 40 & 23 & 17 & 23 & 3 & 10 & 40 & 17 & 57 & $\begin{array}{ll}7 & 10\end{array}$ & 25,8 \\
\hline & $\mathrm{M}$ & 20 & 20 & 50 & 33 & 53 & 23 & 43 & 40 & 20 & 0 & 33 & 27 & 20 & 10 & $\begin{array}{ll}0 \quad 93\end{array}$ & 32,4 \\
\hline & $\mathrm{PO}$ & 0 & 0 & 0 & 0 & 0 & 0 & 0 & 0 & 0 & 0 & 0 & 0 & 0 & 0 & 0 & 0,0 \\
\hline & $\mathrm{V}$ & 53 & 57 & 50 & 47 & 70 & 57 & 63 & 40 & 37 & 27 & 83 & 77 & 3 & 70 & $\begin{array}{l}0 \quad 53 \\
\end{array}$ & 52,4 \\
\hline & S & 0 & 0 & 0 & 3 & 0 & 0 & 3 & 0 & 3 & 0 & 0 & 0 & 0 & 0 & 0 & 0,7 \\
\hline & FO & 37 & 0 & 23 & 0 & 7 & 23 & 20 & 33 & 0 & 27 & 47 & 7 & 0 & 20 & $\begin{array}{ll}0 & 10\end{array}$ & 16,9 \\
\hline
\end{tabular}

mãe-bebê, o que parece ser, por sua vez, determinado pelo contexto social da díade (BORNSTEIN, 1995).

Deve se considerar a existência de acentuadas diferenças individuais entre os bebês já no nascimento, como apontam BRAZELTON, KOSLOWSKI e MAIN ( 1974) e mesmo antes do nascimento, como aponta PIONTELLI ( 1995). Entretanto, parece razoável considerar a existência também de um repertório comum de atividades características de bebês recémnascidos normais, que, pelo próprio nível de desenvolvimento dos bebês, parecem ser restritas em sua variedade. Nesta investigação, foi verificada a predorninancia das seguintes atividades dos bebês: Olhar o ambiente (64,4\%), focalizar (52,4\%), mamar $(32,4 \%)$ e olhar a mãe $(32,2 \%)$. A ativida- de de movimentar membros não foi considerada porque entende-se que os dados obtidas com a classificação dos estados do bebé revelam mais apuradamente a movimentação corporal e o estado de vigília predominante nos diversos intervalos de observação.

Como se pode verificar, ocorreram episódios de interação, em média, em torno de 10\% dos intervalos de observação. Em três, das quinze observações realizadas, não foram identificados episódios de interação.

Predominaram episódios de interação referentes ao domínio social, tendo sido identificada apenas uma interaçao de domínio didatico. Este predomínio pode ser considerado como previsível, em função da idade dos bebês. Como foi observado por BORNSTEIN e TAMIS-LEMONDA 
(1990) e SE1DL DE MOURA e RIBAS (1996) o aumento da ocorrência de domínios didáticos de interação se dá quando o bebê tem em tomo de dois meses de idade. Esta modificação parece ocorrer em função do aumento significativo da exploração do ambiente por parte do bebê, e neste sentido os achados de KAYE e EOGEL (1980) corroboram esta afirmação, indicando um declínio significativo da proporção de tempo durante o qual as crianças mantêm a atenção nas faces das mães, com as idades de 6, 13 e 26 semanas. Por outro lado, também aumenta, por parte da mãe, a iniciativa de estimulação didática, como pôde ser identificado por SEIDL DE MOURA e RIBAS (1996).

A natureza das interações em um período tão precoce do desenvolvimento apresenta peculiaridades, como as limitações do repertório de comportamentos do bebê e também da mãe, na medida em que ela seleciona os meios pelos quais interage com o bebê e a própria limitação dos contextos específicos para a ocorrência das interações. Os contextos específicos que predominaram nos episódios de interação foram os seguintes: bebê no colo da mãe, sem estar mamando (oito episódios), cuidado como: trocar fralda, vestir, dar remédio (oito episódios) e bebê no colo da mãe mamando (sete episódios).

Pode-se considerar que cada um desses contextos envolve a realização de scripts especificos. No caso do contexto específico de interação em que o bebê está no colo da mãe mamando, por exemplo, o script construído culturalmente parece envolver, basicamente, as seguintes atividades da mãe: olhar para o bebe, pegá-lo no colo, colocá-lo numa detemminada posição e dar a ele o seio ou mamadeira e, no caso do bebê, a atividade de mamar. Entretanto, observa-se que, além dessas atividades básicas, outras que não são indispensáveis para que a ação de amamentação ocorra se mostram presentes com frequência: a mãe tende a olhar para o bebê e tocá-lo durante a amamentação. O contexto específico de cuidado, seja ele a troca de fralda ou o banho do bebê envolve a realização de uma série de atividades e a utilização dá uma variedade de artefatos que são característicos do grupo social estudado.

Os episódios de interação observados apresentaram uma duração média de 24 segundos, variando entre 6 segundos e 2 minutos, 48 segundos. Mesino com a faixa de variação observada, pode-se dizer que foram, em geral, curtos os episódios em que mãe e bebê estavam engajados. Esses resultados parecem indicar que são principalmente as características do bebê, como parceiro, que impõem limites temporais à interação.

As durações médias dos episódios de interação variaram nos diferentes contextos da troca: cuidado (9. 5s), bebê no colo, sem estar mamando $(13,25 s)$ e bebê no colo, mamando (53,3 s). Uma interpretação possível para a maior duração dos episódios no contexto da amamentação pode ser a de que a posição em que os parceiros se encontram é facilitadora do maior engajamento, no sentido da manutenção do contato visual. A distância pequena em que os rostos dos dois ficam um do outro também parece propiciar o prolongamento do contato visual, na medida em que bebês da faixa etária estudada nesta pesquisa (22 a 37 dias) enxergam bem alvos distantes de 25 a 30 centímetros conforme apontam KLAUS e Kl.AUS (1989). Outro aspecto que deve ainda ser levado em conta é que a própna atividadade de amamentação tende a durar mais tempo do que outras como trocar fralda, dar banho e vestir a roupa do bebê.

Predominaram as interações pelo contato face-a-face, mas deve ser destacada a ocorrência de dois episódios de interação durante os quais os bebês não olharam para o rosto das mães e um episódio no qual a mãe não olhava diretamente para o rosto do bebê.

A classificação dos estados de vigília do bebê mostrou, considerando todas as diades, uma variação entre os estados 2 e 6 . Dos quinze bebês analisados, os estados predominantes foram os se guintes: em oito bebês predominou o estado 4 (alerta), em quatro bebes o estado 3 (sonolência), em dois bebês predominou o estado 5 (agitado) e em um bebê o predomínio foi do estado 6 (choro). Deste modo a maioria dos bebês encontravase no estado denominado por BRAZELTON \& CRAMER (1992) como alerta passivo.

Como apontam BRAZEI.TON \& CRAMER (op. cit.), os estados de vigília do bebê constituem um dos elementos importantes do contexto. Observou-se, a partir de uma análise qualitativa dos vídeos, que as mães tendem, de fato, a levar em conta a informação acerca do estado do bebê para guiar seu comportamento. A diminuição das atividades de interferência, utilizando-se a terminologia empregada por BRAZELTON \& CRAMER (op. cit.), ou seja, falar com o bebê, vocalizar, tentar chamar a atenção dele, durante a amamentação, pôde ser identificada nas análises realizadas e é um bom exemplo nesse sentido. Outros exemplos encontrados foram: tentativas, por parte da mãe, de manter determinado estado do bebê, quando este começa a alterar seu padrão de movimcntação corporal e de vocalização indicando uma passagem do estado 4 ao 5 . Ou ainda, tentativas de mudar o estado do bebê, durante um contexto de amamentação, no qual as mães, ao verem os bebês passarem do estado 4 ao estado 3 ou 2 , tendem a agir no sentido de trazê-los novamente ao estado 4. 
A análise dos estados dos bebês que predominam durante os episódios de interação e tentativa de interação não efetivada revelou que $80 \%$ destes episódios ocorreram quando os bebês estavam no estado 4, corroborando as afirmações de diversos autores, tais como KLAUS \& KLAUS (1989), de que o estado de alerta passivo é o mais favorecedor para a interação.

Todos os episódios de interação foram classificados como sincrônicos, de acordo com o procedimento adotado, semelhante ao de ISABELLA, BELSKY \& EYE (1989). Mesmo em uma fase tão precoce do desenvolvimento do bebê (idade variando entre 22 a 37 dias), as trocas da díade apresentam esta característica, na medida em que os parceiros pareciam responder um ao outro, manifestando, durante as interações, comportamentos relacionados e coerentes.

Outro aspecto que deve ser ressaltado é a variedade dos ajustes que as mães fazem em seus comportamentos de modo a poder melhor se relacionar com os bebês. Estes incluem: realização de mudanças posturais, como posicionar o rosto na direção, altura e com proximidade ao rosto do bebê, criação de situações facilitadoras de interação, seleção de meios de comunicação mais adequados para se dirigir ao bebê em diferentes contextos, regulação de suas próprias atividades em função dos estados de vigilia do bebê.

Ainda, no que se refere às mães, deve-se destacar o quanto estas se apropriam das ações comunicativas dos bebês, dão a elas um significado, as interpretam de acordo com suas referências, e agem, em resposta aos bebês, de acordo com esta interpretação. As atribuições feitas pelas mães e reveladas em suas falas mostraramse predominantemente vinculadas a preferências e vontades dos bebês. Isto revela uma concepção de que os bebês, desde muito cedo, mostram-se capazes de fazer uma série de discriminações sensoriais e ter, consequentemente, diversas preferências.

Os dados obtidos com a aplicação do $Q C B R$, foram analisados em tennos do escore total. O grupo de quinze mães que respondeu ao questionário obteve um escore total médio de $145,28(\mathrm{dp}=12,16)$. Trata-se de um escore médio alto, compatível com resultados obtidas por SEIDL DE MOURA, RIBAS e RIBAS (op. cit.) no grupo de mulheres e superior ao resultado obtido no grupo de homens. Supõe-se que o escore médio alto, obtido também nesta amostra de mulheres, corrobora a interpretação de que isto se dá em função do processo de socialização das meninas, que são orientadas para assumir papel parental, o que pode levá-las a uma maior atenção e reflexão sobre o assunto. As mães avalia- ram, portanto, de forma bastante positiva e acurada as competências do bebê recém-nascido, considerando-o como um ser ativo e participante das trocas sociais. Isto também se relaciona com seu nível de escolaridade, o que é compatível com as diferenças significativas observadas no estudo que vem sendo citado entre os resultados dos sujeitos de segundo e terceiro graus com os de primeiro grau.

Buscou-se identificar correlações entre os escores totais obtidos com o $Q C B R$ e a percentagem de ocorrência das seguintes atividades da mãe: Falar, Sorrir, Tocar o bebê, Atribuição de significado, e com a variável presenca de interação (expressa em termos de número de episódios identificados e/ou percentagein de ocorrência). Não foram identificadas correlações significativas entre os escores do QCBR e as variáveis interação e atribuição de significado. Foram identificadas correlações significativas entre os escores totais e as seguintes atividades das mães: Falar $\left(r_{s}=0,54 ; \mathrm{P}<0,05\right)$ e sorrir $\left(r_{s}=0,55 ; \mathrm{P}<0,05\right)$.

A identificação de correlações entre falar e sorrir, por parte da mãe, e o escore total no $Q C B R$ mostra uma congruência entre o status que as mães atribuem a seus bebês recém-nascidos e a forma como agem em relação a eles. Parece que quanto mais as mães consideram os bebês competentes, mais lidam com eles como interlocutores e realizam mais frequentemente atividades comunicativas do tipo falar e sorrir.

As atividades das mães e dos bebês, identificadas nos episódios de interação, foram agrupadas de modo a compor uma nova variável que exprime um indice geral de atividade. $\mathrm{O}$ índice de atividade da mãe foi constituído por: falar, olhar o bebê, sorrir, tocar o bebê, vocalizar e atribuir significado e o índice de atividade do bebê foi constituído por: olhar a mãe, mamar, vocalizar, olhar ambiente e tocar a mãe. Buscou-se identificar correlações entre os escores totais obtidas no $Q C B R$ e os índices de atividade da mãe e do bebê. Os resultados indicaram uma correlação significativa entre o escore total obtido no $Q C B R$ e o índice de atividade da mãe $\left(r_{s}=0,62 ; p<0,05\right)$.

Os resultados sugerem que dois componentes do contexto de troca da díade, atividades e representações das mães em relação aos bebês, estão vinculados. Entretanto, parece razoável sugerir que outros dois componentes devem estar associados a estes, são eles: as atividades e os estados dos bebês, na inedida em que as mães parecem se basear nestes elementos para dar significado às ações dos bebês e para regular seu próprio comportamento (HECKHAUSEN, 1987; SEIDL DE MOURA \& RIBAS, 1996). 


\section{CONCL USÕES}

Entende-se que a identificação de um conjunto de atividades comuns a mães e bebês, como parceiros em trocas sociais, e de episódios de interação traz elementos importantes para a discussão acerca da natureza dos primeiros processos interacionais. Os dados obtidos com a observação das díades certamente não representam todas as situações de troca possíveis, mas demonstram a possibilidade de ocorrência de uma variedade de atividades dos parceiros e de episódios de interação e tentativa de interação não efetivada em detenninado contexto.

Evidências de um estudo longitudinal anterior foram confinnadas, em relação à presença de interações em etapas inicias do desenvolvimento infantil. Corroborando pesquisas anteriores, BORNSTEIN \& TAMIS-LEMONDA (1990) e SEIDL DE MOURA \& RIBAS (op. cit.), foi possível identificar principalmente episódios de interação de dominio social, ou seja, centrados nas atividades da própria díade e não mediados por objetos e eventos do mundo. Isto indica a necessidade inicial de regulação da ação conjunta para, em seguida, ser possível interagir em torno de objetos e chegar a atividades mediadas conjuntas.

Os dados obtidos com o QCBR confirmaram resultados anteriores que indicam que mulheres têm escores mais altos que homens neste instrumento, independenternente de terem ou não filhos. Revelaram, ainda, que as mães avaliam de forma bastante positiva e acurada as competências do bebê recém-nascido, considerando-o como um ser ativo e participante das trocas sociais. Apesar de não ter sido identificada correlação significativa entre os escores do QCBR e a variável interação, pôde ser identificada correlaçao signi- ficativa entre o indice de atividade da mãe e as variáveis falar e sorrir isoladamente, com o escore total do QCBR. Mostrou-se, portanto, uma coerência entre o status que as mães atribuem a seus bebês recém-nascidos e a forma como agem em relação a eles.

Pode-se considerar que houve algum avanço em relação ao esforço de articulação teórica, na medida em que os dados obtidos com esta pesquisa estão contribuindo para fortalecer o conhecimento acerca do contexto (e. g. atividades dos parceiros, estados dos bebês, interações, tentativas de interação, representaçoes das mães acerca dos bebês) no qual se dá o desenvolvimento cognitivo. Ainda neste sentido, tem sido possível vincular as noções Zona de Construção e de Mentes Interativas aos achados da pesquisa. Os dados podem ser interpretados como evidenciando proto-negociações de significado. O bebê age e a mãe atribui significado às suas atividades, agindo em função da interpretação feita. A linguagem é utilizada apenas pela mãe, mas se pode considerar que, como o discurso, as ações e seu sentido são, para os parceiros destas diades, indeterminadas. A partir das características específicas de ambos, estabelece-se um processo de comunicação entre mãe-bebê, que permite a regulação mútua dos seus comportamentos. Hipotetiza-se que é nessas trocas, principalmente, que se dá a construção do conhecimento, por parte do bebê, acerca dos objetos, pessoas e eventos do mundo.

Desta forma, a análise dos dados parciais desta pesquisa permitiu a continuidade das reflexões teóricas que vêm sendo feitas no sentido de construção de um modelo que dê conta de evidências de diferentes vertentes de investigação e explique a ontogênese do desenvolvimento de forrna inseparável do contexto social.

\begin{abstract}
This work is part of a line of investigations that has been trying to articulate the sociocultura] perspective with results of two other research arcas: infant development and adultbaby interactions. In this sense, an integration has been promoted between evidences on motherini ‘ant interactions and theoretical studies regarding the notions of construction zone and of interactive minds. Ideas about the notion of context as a space where such interactions take place and t'rom which they can be better understood, or development niches, have been articulated. Elements that constitute the contexts have been identified (e.g. scenes, scripts, formats, partners, their respective activities and representations and the use of artifacts of different leveis). In this work, contexts in which interactions between newborn babies and their mothers are established were examined. Scenes, scripts, the partners' activities, interaction and episodes of interaction attempts were identified. The relation between the mothers' conceptions concerning the babies' competence and the nature of their activities and interactions with the babies were also investigated. For such, an observation methodology and a questionnaire developed by the research group were used (QCBR - questionnaire lòr the evaluation of the adults' conception concerning the newborn baby's competencies). So far 15 dyads of mothers and babies, with ages between 22 and 37 days, were observed and filmed for 20 minutes in their residences. A significant correlation between the index of the mother's activity and the total score in the QCBR was identified. The present
\end{abstract}


work did not identify a correlation between total score in the QCBR and the variable interaction. The present work can contribute to a better understanding of cognitive development as a process inserted in the social context.

Key-words: mother-infant interaction, early child development, conceptions of development.

\section{REFERENCIAS BIBLIOGRÁFICAS}

BALTES, P.; STAUDTNGER, U. M. Interactive minds in a life span perspective: prologue. In: BALTES, P.; STAUDINGER, U. M. Interactive minds. Life span perspectives on the social foundations of cognition. Cambri-dge, etc., Cambndge University Press, 1996. p.1-32.

BERTENTHAL, B. I. Origins of early development of perception, action, and representation. An. Rev. of Psych.,47: 431 -459,1996.

BORNSTEIN, M. Forrn and function: implications for studies of culture and human development. An. Rev. of Psych. 1 1(1): 123137, 1995.

BORNSTEIN, M.; l'AMIS-LEMONDA, C. S. Activihes and interactions of mothers and their firstborn infants in the tirst six months of life: covariation, stability, continuity, correspondence and prediction. Child Dev., 61: 1206$1217,1990$.

BRAZELTON, T. B.; CRAMER, B. G. As priineiras reações. São Paulo, Ed. Martins Fontes, 1992.

BRAZELTON, T. B; KOSLOWSKI, B.; MAIN, $M$. The origins of reciprocity: The early mother-infant interaction. In: LEWIS, M.; ROSENBLUM, L. The effect of the infant on its caregiver: New York, etc., John Wiley \& Sons. 1974. p. 49-76.

BRUNER, J. The culture of education. Cambridge, etc., Harvard University Press, 1996.

BRUNER, J. In search of mind. Essays in autobiography. New York, etc., Harper \& Row, 1983.

COLE, M. Interacting minds in a lífe-span perspective: a cultural-historical approach to culture and cognitive development. In: BALTES, P; STAUDINGER, U. M. Interactive minds. Life span perspectives on the social fòundations of 'cognition. Cambridge, etc., Cambridge University Press, 1996. p. 59-87.

GOOONOW, J. J. Parents' ideas, actions, and feelings: models and methods from developmental and social Psychology. Child Dev., 59: 286-320, 1988.

HECKIIAUSEN, J. How do mothers know? Infants' chronological age or infants' performance as determinants of adaptation in maternal instruction? J. of Exp. Child Psych., 43: 212-226, 1987.
ISABELLA, R. A.; BELSKY, J.; EYE, A. Origins of infant-mother attachment: an examination of interactional synchrony during the infant's first year. Dev. Psych., 25(1): 12-21, 1989.

KALINS, I; BRUNER, J. The coordination of visual observation and instrumental behavior in early Infancy. Perception, 2: 307-314, 1973.

KLAUS, M.; KLAUS. P. O surpreendente recémnascido. Porto Alegre, Ed. Artes Médicas, 1989.

KAYE, K.; FOGEL, A. The temporal of structure of face to-face communication between mothers and infants. Dev. Psych., 16(5): 454464, 1980.

LEBOVICI, S. O bebê, a mãe e o psicanalista. Porto Alegre, Ed. Artes Médicas, 1987.

MILLER, S. A. Parents' beliefs about children's cognitive development. Child Dev., 59: 259285, 1988.

NEWMAN, D.; GRIFFIN, P.; COLE, M. The construction zone. Working for cognitive change in selection. Cambridge, etc., Cambridge University Press, 1989.

PAPOUSER, H. From adaptative responses to social cognition: the learning view of development. In: BORNSTEIN, M. H.; KESSEN, W. Psychogical development from infance. Image for atention. Hillsdale, Erlbaum, 1979. p. 251-267.

PIONTELL1, A. De feto a criança. Rio de Janeiro. Ed. Imago, 1995.

RIBAS, R. C. Considerações sobre os modelos de desenvolvimento cognitivo de J. Piaget e L. S. Vygotsky. Arq. Bras. Psic., 45(3/4): 137149, 1993.

RIBAS, A. F. P. Interações precoces mãe-bebê: A gênese de zonas de construcão. Rio de Janeiro, 1996. [Dissertação de Mestrado - Universidade Federal do Rio de Janeiro].

RIBAS, A F. P.; SEIDL DE MOURA, M. L. Construcão de um Questionário para o estudo da concepção de adultos acerca das competências do recém-nascido. Arq Bras. Psic., 47(2): 89-99. 1995.

SCAIFE, M.; BRUNER, J. S. The capacity for joint visual attention in the infant. Nature, 253: 265-266. 1975.

SETDL DE MOURA, M. L. Interaçao social e desenvolvimento. A concepção de adultos do bebê reccem-nascido. Projeto de Pesquisa, Rio de Janeiro: UERJ, 1995. 
SEIDL DE MOURA, M. L.; RIBAS, A. F. P. Mother and infant interaction: The genesis of zones of proximal development. In: CON-FERENCE FOR SOCIOCULTURAL RESE-ARCH, $2^{\circ}$, Genève, 1996. Abstiracts, Genève, 1996. p. 45.

TREVARTHEN, C.; HUBLEY, P. Secondary intersubjectivity: confidence, comfiding and acts of meaning in the fiest year. In: LOCK, A. Action gesture and symhol. The emergence language. London, etc., Academic Press, 1978. p. 183-229.
WERTSCH,.J. V.; DEL R10, P.; ALVAREZ, A. Sociocutural studies: history, action and mediation. In: WERTSCH, J. V.; DEL RIO, P; ALVAREZ, A. Sociocultural Studies of Mind. Cambridge, Cambndge University Press, 1995. p. I1-34.

VIBBERT, M.; BORNSTEIN, M. H. Specific associations between domains of mother-chlild interaction and toddler referential language and pretense play. Inf. Beh. Dev., 12: 163-184, 1989. 